\section{El síndrome de abstinencia de la marihuana}

A pesar de que los defensores de la marihuana la describen como una droga suave y poco peligrosa, los diversos estudios científicos que se han realizado desde los años setenta indican lo contrario. La nueva investigación subsidiada por el Instituto Nacional de Investigación sobre el Abuso de Drogas en la Escuela de Medicina de Harvard es una prueba más de que los usuarios crónicos de marihuana sufren del síndrome de abstinencia cuando dejan de fumar la sustancia. El comportamiento agresivo es parte importante de este síndrome, caracterizado también por insomnio, agitación, pérdida del apetito e irritabilidad. Si bien el síndrome es menos pronunciado que el de la abstinencia del alcohol, los opiáceos o la cocaína, es suficientemente fuerte para motivar la reincidencia en el hábito. Es esencial llegar a un mejor conocimiento de la naturaleza de ese síndrome para poder desarrollar estrategias contra el uso de la marihuana, que es la droga ilícita usada más comúnmente en los Estados Unidos de América. En la encuesta de hogares sobre el uso indebido de drogas que se llevó a cabo en 1997, más de 11 millones de personas declararon que habían fumado marihuana durante el mes anterior. Esta droga no solo causa adicción y un consumo compulsivo que afecta a las relaciones del usuario con la familia y su desempeño en la escuela o el trabajo, sino que causa problemas físicos, como una tos crónica relacionada con el hábito de fumar o problemas psicológicos por el efecto de sedación que tienen las dosis altas.

En el estudio de Harvard se usó una prueba computadorizada de comportamiento agresivo para comparar a 17 usuarios crónicos de marihuana con 20 fumadores infrecuentes o que habían dejado el hábito. Todos los participantes eran voluntarios que se abstuvieron del uso de marihuana durante el estudio. Ninguno sabía que se estaba observando su comportamiento, pues se les hizo creer que el estudio estaba centrado en medir características fisiológicas como el pulso y la temperatura. Se trataba de un juego en el que se intentaba ganar puntos (cada punto valía 50 centavos) y restarle los tantos ganados a un adversario que se suponía estuviera participando desde otra habitación. Los sujetos participaron al comienzo del estudio y en los días 1, 3, 7 y 28 de abstinencia. Los resultados muestran claramente que los usuarios regulares de marihuana mostraron mucha más agresividad que los del otro grupo en los días 3 y 7, lo que indica su irritabilidad durante la primera semana de abstinencia. (National Institute on Drug Abuse. Chronic marijuana users become aggressive during withdrawal. NIH News Release. Sin fecha.)

\section{La propaganda del cigarrillo sigue atrayendo a jóvenes fumadores}

La mitad de los adultos que fuman se inician en el hábito antes de los 15 años y $90 \%$, antes de los 18. Los que no empiezan a fumar antes de los 21 años tienen buenas probabilidades de nunca sentirse atraídos por el tabaco. En los Estados Unidos, las encuestas nacionales revelan que cerca de $30 \%$ de los chicos de 12 a 14 años ya han probado los cigarrillos. Lamentablemente, después de años de disminución del tabaquismo, la prevalencia ha vuelto a aumentar entre los estudiantes de escuela secundaria. Los anuncios promulgados por los fabricantes son un elemento crítico en el éxito que tiene la industria para reclutar a fumadores jóvenes. Las compañías tabacaleras gastan enormes sumas de dinero (US\$ 4600 millones en 1991) en popularizar sus productos $\mathrm{y}$, a pesar de que la propaganda en revistas ha disminuido, se ha mantenido estable en las que cuentan con grandes números de lectores adolescentes. Varios estudios muestran que los adolescentes son muy susceptibles a esa propaganda y, en particular, a la que se ha ideado específicamente para su grupo de edad. Respondiendo a fuertes críticas del público, en 1965 el Instituto del Tabaco, asociación comercial que representa a las seis más grandes compañías tabacaleras de los Estados Unidos, puso en ejecución un código voluntario que restringe la promoción y propaganda de los cigarrillos. La versión de 1990 del código proscribe cualquier sugerencia de que el hábito de fumar es esencial para lograr prominencia social, distinción, éxito o atracción sexual; prohíbe mostrar a una persona que fuma exageradamente; permite incluir en los anuncios a personas sanas y atractivas siempre que no se sugiera que su atractivo se debe al hábito de fumar; prohíbe relacionar el tabaquismo con el talento atlético o la fuerza física; y exige que los modelos que aparecen en los anuncios aparenten más de 25 años de edad.

Dado que la prevalencia del tabaquismo en los adolescentes ha aumentado de nuevo, se llevó a 
cabo una investigación para determinar si la propaganda actual se adhiere a las restricciones del código. Se encuestó a una muestra representativa de 913 escolares de 10 a 15 años de edad del estado de Massachusetts mediante un cuestionario autoadministrado. Se les enseñaron anuncios de cigarrillos y de varios otros productos de sus 15 revistas favoritas para saber qué impresión recibían de ellos. Los anuncios elegidos contenían figuras humanas. Según las respuestas de la mayoría, los anuncios daban a entender que los cigarrillos traían popularidad, éxito, atracción sexual, y hasta salud. También opinaron que los modelos que aparecen en los anuncios estaban fumando de forma exagerada y en general tenían menos de 25 años. El estudio muestra que el código ha fallado, pues los anuncios actuales todavía comunican mensajes culturales positivos a los jóvenes sobre el hábito de fumar. Posiblemente cualquier anuncio que muestre seres humanos o caracteres antropomórficos comunicará inevitablemente mensajes positivos. Se concluye que, por esa razón, es necesario introducir y evaluar nuevas políticas públicas que aborden los problemas causados por las imágenes de propaganda. (Barbeau EM, DeJong W, Brugge DM, Rand WM. Does cigarette print advertising adhere to the Tobacco Institute's voluntary advertising and promotion code? An assessment. J Public Health Policy 19(4):1998.)

\section{La apnea del sueño y el riesgo de accidentes de tránsito}

En España, los accidentes de tráfico cuestan anualmente de 1 a $2 \%$ del producto nacional bruto. En los Estados Unidos, la Comisión Nacional de Investigación sobre los Trastornos del Sueño estimó que en 1988 los accidentes relacionados con la somnolencia del conductor costaron de US $\$ 43000$ a 56000 millones. De hecho, la Comisión determinó que el elemento de somnolencia puede estar implicado en 36\% de los accidentes de tráfico que causan muertes y en 42 a $54 \%$ de todos los accidentes en general. Se han llevado a cabo investigaciones con simuladores sobre los efectos de la apnea del sueño en la capacidad para conducir, en los que se demostró una reducción de la concentración, mayor lentitud en el tiempo de reaccionar y recurrencia de períodos de sueño. Otros análisis retrospectivos mostraron que las personas con apnea del sueño habían tenido un número de accidentes de tráfico de dos a tres veces más alto que el resto de la población.

Un estudio español de casos y controles realizado en 1995 ha corroborado la influencia de ese trastorno del sueño en los accidentes de tráfico. En el estudio se incluyeron como casos 102 pacientes que habían recibido atención de urgencia en hospitales de las ciudades de Burgos y Santander, España, como resultado de accidentes de vehículos de motor. Los controles fueron 152 pacientes elegidos al azar de centros de atención primaria en las mismas ciudades y apareados con los casos por sexo y edad. Para detectar la presencia de apnea, los pacientes se tamizaron en sus hogares mediante poligrafía de la respiración y el diagnóstico fue confirmado por polisomnografía convencional. Para cada participante se calculó un índice de apnea-hipopnea, basado en el total de episodios de apnea e hipopnea dividido por el número de horas de sueño.

Los participantes tenían una mediana de edad de 44 años y 77\% eran varones. Comparados con los que no sufrían apnea del sueño, los pacientes con índices de 10 o más episodios de apnea-hipopnea tuvieron una razón de posibilidades de 6,3 (IC95\%: 2,4 a 16,2) de tener un accidente de tráfico. Esta relación se mantuvo después de ajustar por factores de confusión potenciales tales como el consumo de alcohol, trastornos de refracción visual, índice de masa corporal, años de conducir, edad, antecedentes de accidentes de tráfico, uso de medicamentos que causan somnolencia y horas de sueño habituales. Entre los que tenían índices de apnea-hipopnea de 10 o más, el riesgo de accidentes aumentaba si habían consumido alcohol, aun en pequeñas cantidades, el día del accidente. Cabe notar que se había excluido de la investigación a los conductores contra quienes había procesos jurídicos por conducir bajo la influencia del alcohol. Según este estudio, la asociación entre la apnea del sueño y el riesgo de accidentes de tráfico es aun más fuerte de lo que se había pensado. (Terán-Santos J, Jiménez-Gómez A, Cordero-Guevara J, et al. The association between sleep apnea and the risk of traffic accidents. N Engl J Med 1999;340:847-851.)

\section{La atención de salud primaria exige el desarrollo de la comunidad}

Como parte esencial de la reforma de la atención de salud en el Reino Unido, se han formado los llamados grupos de atención primaria (o grupos locales de salud, en Gales). Esos grupos tienen la responsabilidad de modelar los servicios, evaluar las necesidades de salud, reducir las faltas de equidad en los servicios, escuchar las opiniones de los usuarios $\mathrm{y}$ trabajar en conjunto con las agencias locales. Todo ello exige una gama de habilidades que pocos profesionales de la salud tienen actualmente, y el gobierno no ha proporcionado los medios conceptuales o gerenciales ni la infraestructura financiera para la participación del público. Las sesiones públicas constituyen el único mecanismo de consulta 
reconocido oficialmente, pero no son una manera adecuada de involucrar a las comunidades en los problemas de salud que las afligen, especialmente cuando se trata de grupos marginados. Una solución es que los prestadores de atención primaria trabajen junto con los proyectos de desarrollo comunitario, ya que estos hace años se ocupan de esos asuntos. En el desarrollo comunitario se reconocen las causas sociales, económicas y ambientales de la mala salud y se combina la participación de los usuarios con la de los proveedores oficiales de los servicios para mejorar la salud y reducir las faltas de equidad. Las comunidades pueden constituirse geográficamente como vecindarios o por intereses de grupos de usuarios. Es preciso contar con trabajadores entrenados en el desarrollo de comunidades para que reúnan a la gente local con objeto de:

- identificar y apoyar las redes comunitarias que ya existen para mejorar la salud;

- determinar las necesidades de salud con especial atención a los grupos marginados y a los que sufren por desigualdad de oportunidades;

- trabajar con otras agencias pertinentes, incluso grupos de la comunidad, para abordar las necesidades sentidas; y

- estimular el diálogo con los sistemas oficiales para lograr servicios más apropiados y asequibles.

Se ha verificado que el apoyo de la comunidad expresado en las redes sociales mejora la salud y que la confianza mutua y la cohesión de los miembros de un grupo contribuyen a reducir la mortalidad. Por el contrario, la falta de control, de autoestima y de apoyo social aumentan la morbilidad. Además, la evaluación de necesidades enfocada en la comunidad no solo da con los problemas, sino a veces con las soluciones. En la localidad de Lewisham, por ejemplo, la evaluación llevó a crear un puesto de asesor de la juventud y, como resultado, han mejorado considerablemente los conocimientos de anticoncepción y salud sexual. Las prácticas de atención también se han modificado para tener más en cuenta las necesidades de los jóvenes. En St. Peter's Ward, una zona de bajos ingresos de Plymouth, el enfoque de desarrollo de la comunidad ha permitido poner en ejecución un proyecto local de pruebas de embarazo gratuitas y recursos comunitarios para la instrucción y el apoyo de los futuros padres. Este enfoque puede igualmente reducir el impacto de la pobreza en la salud. En Torquay existe ahora una cooperativa, administrada por gente local, que provee alimentos sanos y nutritivos a precios bajos. En la misma comunidad se ha establecido un grupo de seguridad que ha conseguido cambios y mejoras en las viviendas, las áreas de juego para los niños, y los servicios de policía. En Bradford se aumentó el tamizaje del cáncer de mama y cervicouterino en mujeres de minorías étnicas marginadas. Es muy importante saber que mediante la continuación del desarrollo de la comunidad, la unión de grupos comunitarios y las iniciativas de las autoridades locales, una agencia puede apoyar o desafiar el planeamiento del grupo de atención primaria. (Fisher B, Neve H, Heritage Z. Community development, user involvement, and primary health care [editorial]. BMJ 1999;318:749-750.)

\section{Impacto de la disponibilidad de preservativos en escuelas de Seattle}

En un intento por reducir los embarazos indeseados y las enfermedades de transmisión sexual incluida la infección por VIH, para principios de 1995 casi 400 escuelas secundarias de los Estados Unidos habían iniciado programas para que los estudiantes pudieran obtener preservativos de máquinas, canastas o la clínica estudiantil. Cuesta poco ejecutar estos programas que se esperaba tuvieran resultados beneficiosos. Desde entonces se habían hecho solo tres estudios sobre el uso de los preservativos y sus efectos en el comportamiento sexual y anticonceptivo de los estudiantes. En dos de los estudios se encontró un aumento significativo del uso de los preservativos pero no de la actividad sexual. Sin embargo, los estudios mencionados estaban limitados por falta de datos de base y grupos de comparación, muestras de población demasiado pequeñas y dificultades de seguimiento. Además, dos de los estudios medían el impacto de programas de mayor amplitud para prevenir la infección por VIH o promover la salud y no solo el de la disponibilidad y uso de preservativos. Por lo tanto, se realizó un estudio dedicado exclusivamente a determinar el número de preservativos obtenidos por los estudiantes de 10 escuelas de la ciudad de Seattle y a analizar los cambios que se produjeron con el tiempo en su comportamiento sexual y el uso de condones.

En 1993, cinco escuelas secundarias con centros de salud empezaron a ofrecer los preservativos en varias canastas localizadas en el área de recepción, el área de examen físico y los baños del centro de salud. Cerca de las canastas se colocaron impresos con información sobre la abstinencia, el uso de condones, y la prevención del VIH. Los estudiantes podían obtener por su cuenta todos los preservativos que quisieran, cada vez que quisieran, sin permiso de los padres ni asesoramiento previo. Un año más tarde se instalaron una o dos máquinas de vender preservativos en lugares transitados de cada una de las cinco escuelas que no tenían centros de 
salud y en dos de las escuelas con centros. Los preservativos costaban 25 centavos cada uno y se podían comprar sin ninguna restricción. Cerca de cada máquina se colocaron folletos informativos pertinentes. En 1995 dos de estas escuelas habían abierto clínicas y comenzaron también a ofrecer condones en canastas.

Antes de hacer disponibles los preservativos, todas las escuelas habían puesto en marcha programas de educación sexual en clases de salud de 6 meses de duración para los estudiantes de noveno grado. El plan de estudios comprendía información sobre el VIH y el sida y actividades para enseñarles a resistir las presiones de su grupo social, formas de cambiar las normas del grupo, ejercicios para aprender a expresar sus convicciones con soltura y material que mostraba explícitamente el uso apropiado de los preservativos. En la primavera de 1993, se administraron cuestionarios a los estudiantes antes de hacer disponibles los condones y en la primavera de 1995 se llevó a cabo otra encuesta después de ofrecerlos en las escuelas. Los cuestionarios investigaban específicamente el comportamiento sexual y el uso de condones de los estudiantes. Esta segunda encuesta reveló que 29\% de todos los estu- diantes habían obtenido por lo menos un preservativo, pero solo $13 \%$ habían usado en sus relaciones sexuales un preservativo obtenido en la escuela. Los porcentajes eran más altos entre los estudiantes con mayor experiencia sexual (47 y 30\%, respectivamente). De los estudiantes que habían obtenido condones, $62 \%$ habían usado de 1 a $5 ; 18 \%$ usaron de 6 a 10; 8\% usaron de 11 a 20; y 12\%, 21 o más. Entre los estudiantes con más experiencia sexual, los de escuelas con centros de salud usaron los condones obtenidos en las escuelas dos veces más que los que asistían a escuelas con solo máquinas automáticas (42 y 18\%, respectivamente).

El análisis de todos los datos mostró que los estudiantes obtuvieron en promedio 4,6 condones al año, la mayor parte de canastas y muy pocos de máquinas. La disponibilidad no se tradujo en un uso más frecuente de condones. El porcentaje de estudiantes que había tenido relaciones sexuales se mantuvo estable después de iniciado el programa. Este no aceleró la iniciación sexual de los estudiantes y la actividad sexual declinó levemente en las escuelas de Seattle. (Kirby D, Brener ND, Brown NL, Peterfreund N, Hillard P, Harrist R. The impact of condom distribution in Seattle schools on sexual

ERRATA

Frazão y Castellanos, "La participación del personal auxiliar de odontología en los sistemas locales de salud" (Rev Panam Salud Publica/Pan Am J Public Health 1999;5(2):106-115)

Se ruega a los lectores tomar nota de un error en la nota al pie del cuadro que aparece dentro del anexo 1 (p. 114).

- La nota dice que "las cifras representan el número de actividades realizadas en un mes", cuando debe decir que representan el número de actividades realizadas en una hora. 